\title{
Rock stability analysis - A case study
}

\author{
A. Lahmili ${ }^{1}$, L. Ouadif ${ }^{1}$, A. Akhssas ${ }^{1}$ and L. Bahi ${ }^{1}$ \\ ${ }^{1}$ Université Mohammed V, Ecole Mohammadia d'Ingénieurs, Laboratoire de Géophysique Appliquée, de Géotechnique \\ et de Géologie de l'Ingénieur et de l'environnement, Rabat, Maroc
}

\begin{abstract}
The problems of the stability of the mineral-bearing structure ST2 at $560 \mathrm{~m}$ of depth in the east zone of Bou-azzer mine disturbs the advance of the exploitation. The geological and structural study based on field observations and the analysis of core drilling shows the presence of altered and fractured diorite surmounted by cobalt mineralization. Based on the empirical methods of Barton (Q-system) and Bieniawski (RMR) the bed rock is classified as poor quality. The analytical study made it possible to dimension supporting by bow-pieces and bolting. The existence of several types of discontinuities (fault, diaclases and joints) has made the realization of numerical simulation by the finite elements method very difficult. These discontinuities create a network of natural fractures which cut out the blocks in various forms likely to be detached or slip into the excavation, thus encouraging the infiltration of water creating pressure on the massif. The classical studies show their limits in practice for installation of supporting because they must take into account the characteristics of discontinuities. Hence a structural analysis of the massif is essential. The cracking survey of ST2 at $560 \mathrm{~m}$ of depth in the east zone of Bou-azzer mine at $560 \mathrm{~m}$ of depth, and their processing by the DIPS software, showed the existence of three main families of discontinuity NW-SE with a dip of 75SW, NS subverticale and NE-SW with a dip of 57NW, and two families of minor joints NW-SE and NE-SW with successive dips of 40SW and 75SE. The analysis of fracturing surveys allowed us to evaluate the risks of falling blocks and the families of discontinuity responsible for them, and to limit the zones presenting a risk of slip and the families responsible for them. The importance of this study is of knowing how and where to put supporting to be opposed to the risk of fall and tilting of the blocks, caused by the network of discontinuities of the massif.
\end{abstract}

Résumé : Les problèmes de la stabilité de la mine de Bou-azzer zone Est au niveau de la structure minéralisée ST2 à $560 \mathrm{~m}$ de profondeur perturbe l'avancement de l'exploitation. L'étude géologique et structurale basées sur les observations de terrain et l'analyse des sondages carottés, montre la présence de la diorite altérée et fracturée surmontée de la minéralisation de Cobalt constituée de Skuttérudite et la Safflorite, et parfois Loellingite qui entre en contact avec la serpentine. En se basant sur les méthodes empiriques Barton (Qsystème), ou Bieniawski (RMR) le terrain est classé de qualité médiocre, l'étude analytique a permis de dimensionner le soutènement par cintres et boulons d'encrages. L'existence de plusieurs type de discontinuités (faille, diaclases et joints) ont rendu la réalisation de simulation numérique par la méthode des éléments finis très difficile, ces discontinuités créent un réseau de fractures naturelles qui découpent les blocs en différentes formes susceptibles de se détacher ou de glisser dans l'excavation, favorisant ainsi l'infiltration des eaux créant une pression sur le massif. Les études classiques entamées précédemment montrent leurs limites en pratique pour la pose du soutènement, parce qu'ils doivent prendre en considération les caractéristiques des discontinuités. D'où une analyse structurale du massif s'avère indispensable. Les levées de la fissuration au niveau -560 dans la ST2 de la mine de Bou-azzer Est, et leurs traitement par le logiciel DIPS, a montré l'existence de trois familles de discontinuité principales NW-SE avec un pendage de 75SW, N-S subverticale et NE-SW avec un pendage de 57NW et deux familles de joints mineurs NW-SE et NE-SW avec des pendages successive de $40 \mathrm{SW}$ et $75 \mathrm{SE}$. Le traitement des relevées de la fracturation nous a permis d'évaluer les risques de chute de blocs et les familles de discontinuité qui en sont responsables, et de limiter les zones présentant un risque de glissement et les familles qui en sont responsable. L'importance de cette étude est de savoir comment et où mettre le soutènement pour s'opposer au risque de chute et de basculement des blocs, provoqué par le réseau de discontinuité du massif. 


\section{Introduction}

La minéralisation cobaltifères dans la mine de Bou-azzer se présente essentiellement sous forme de filons à fort pendage et de puissance variable qui se développent dans les serpentines au contact avec les diorites quartziques du Précambrien II inférieur. L'exploitation est régulièrement confrontée à des problèmes de stabilité de terrain, ce qui présente évidemment un risque pour la sécurité du personnel et du matériel, et un impact négatif sur la productivité des chantiers.

Dans la présente étude nous allons caractérisé les massifs rocheux de la ST2 de la mine de Bou-azzer Est, au niveau $-560 \mathrm{~m}$ pour le choix du mode de soutènement approprié, en utilisant des méthodes empiriques comme les classifications de Deer, Hoek et Brown, de Barton, de Bienswiski et L'AFTES, complétée par une étude de la fracturation pour évaluer les risques de basculement de blocs et le risque de glissement et limiter les familles de fractures qui en sont responsable.

\section{Contexte géologique}

\subsection{Situation géographique}

Le district de Bou-azzer, se situe dans L'Anti-Atlas central dans la boutonnière précambrienne de Bou azzerEl Graara, à 140 kilomètres au sud-ouest d'Ouarzazate (figure 1).

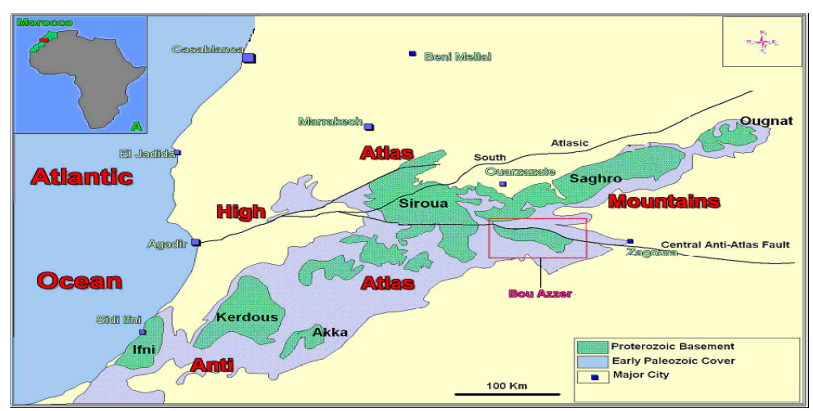

Fig 1: Situation géographique de la boutonnière de Bou-Azzer El Graara [1]

\subsection{Géologie régionale}

La boutonnière de Bou-azzer El Graara est formée essentiellement d'un socle d'âge Protérozoïque et d'une couverture Infracambrienne (figure 2). Une série ophiolitique encaisse principalement les minéralisations cobaltifères et chromifères.

Précambrien I : constitué de gneiss, d'amphibolites, de migmatites et de leucogranites déformés et polymétamorphiques ayant subi plusieurs phases tectoniques (éburnéenne, panafricaine)

Précambrien II représenté par une série volcanosédimentaire de Tachdamt-Bleida, et un complexe ophiolitique de 4 à $5 \mathrm{Km}$, auquel sont liées les minéralisations d'arséniures de cobalt et nickel de Bou azzer Est du précambrien II inférieur, et la série de Tiddiline à dominance de matériaux détritiques formés de molasses, arkose, de la carapace d'altération météorique discontinue d'Ambed qui repose sur le substratum serpentineux du Précambrien II supérieur.

Infracombrien : La couverture Infracambrienne repose en discordance sur le socle. Elle comprend à sa base une puissante série volcano-sédimentaire (formation d'Ouarzazate d'âge néo-protérozoïque supérieur ou PIII) bien stratifiée et subhorizontale, constituée par des andésites, des rhyolites avec beaucoup d'ignimbrites. Les laves intercalées dans cette série sont de nature calcoalcaline surmontée par une série sédimentaire grésodolomitique, dont la puissance ne dépasse pas $1000 \mathrm{~m}$ dans la région de Bou-azzer.

La tectonique postérieure au PIII est de style cassant. C'est l'orogenèse hercynienne, qui a fait rejouer verticalement et horizontalement des blocs du socle et donnent naissance, dans la couverture, à des plis disharmoniques et parfois chevauchants.

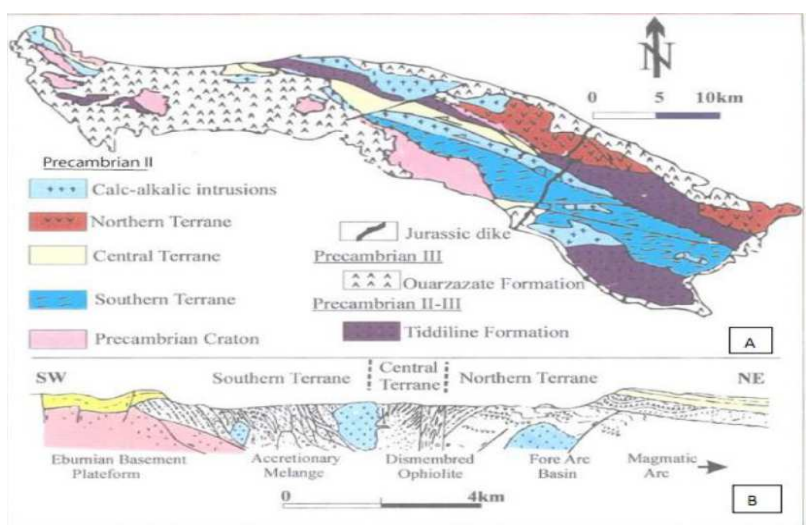

Fig 2: Carte géologique de la boutonnière de Bou Azzer, [2].

\subsection{Géologie du chantier Bou-Azzer Est}

Le gisement de Bou azzer Est, actuellement en exploitation, est situé à environ $2 \mathrm{Km}$ à l'Est du centre minier de Bouazzer. Le contexte géologique local du secteur est caractérisé par l'affleurement des terrains du Précambrien II inférieur, représentés par les serpentines en contact avec les diorites quartziques. La structure ST2 orientée NE-SW et subvertical, actuellement exploitée a une extension moyenne de $160 \mathrm{~m}$ de longueur et une puissance qui varie entre $0.5 \mathrm{~cm}$ et $2.7 \mathrm{~m}$, est reconnue jusqu'à une profondeur de $-560 \mathrm{~m}$.

\section{Etude Géotechnique du massif de la ST2}

Au niveau $-560 \mathrm{~m}$ la minéralisation de la structure ST2 est de direction NW-SE avec un pendage de $60^{\circ}$ vers le NE. Les serpentines constituent le toit et les diorites le mur (figure. 3). L'étude géologique montre que le sens du pendage des serpentines a changé entre les niveaux $510 \mathrm{~m}$ et $-560 \mathrm{~m}$, et la schistosité qui les affectent a pendage fort vers le $\mathrm{SE}$, ce qui a engendré des problèmes d'éboulements dans les premières phases d'exploitation du niveau $-560 \mathrm{~m}$. 


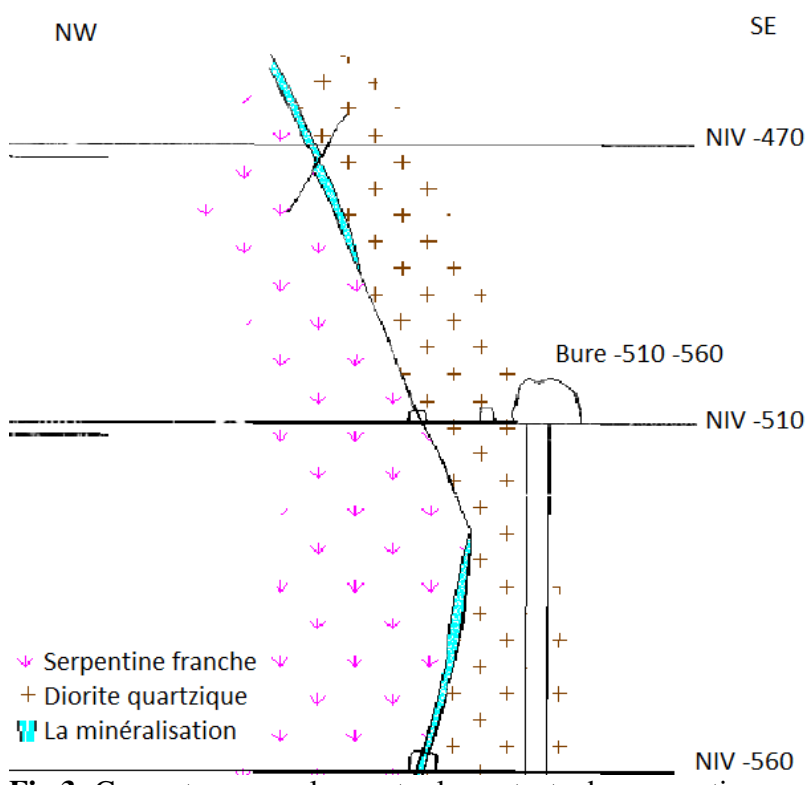

Fig 3: Coupe transversale montre le contacte des serpentines avec les diorites la minéralisation se trouve au niveau du contact.

\subsection{Identification des roches}

Quatre sondages carottés ont été réalisé à partir du niveau -440m jusqu'au $-720 \mathrm{~m}$ pour caractériser le massif rocheux de la ST2, et ont permis d'identifier les différents facies et de localiser la minéralisation.

Il ressort que les infiltrations sont remarquables, des petits blocs chutent facilement lors du purgage. Les serpentines sont friables schistosées, les diorites sont tendres et fracturés, les chlorites témoignent de l'existence de l'altération hydrothermal et les discontinuités existantes sont de différentes types (faille, diaclases, fentes).

\section{rocheux. \\ 2.2 Classifications empiriques du massif}

Les facies concernés par les classifications basées sur approche empirique, sont les Diorites quartziques altéré chloritisées, Diorites quartziques altérées chloritisés fracturées, Diorites quartziques très fracturées, Serpentine franche et Caisse minéralisée avec chlorite.

\subsubsection{Classification de DEERE : RQD (Rock} Quality Designation) [3]

Basée sur l'indice RQD, Deere classe les massifs rocheux de très médiocre à excellent

Le calcule de la moyenne des RQD pour chaque faciès, en utilisant les sondages, nous a permis de classer le massif de la ST2 selon Deer de faible (tableau 1)

Tableau 1. Classification de Deere (RQD)

\begin{tabular}{|l|l|l|}
\hline Roche & RQD & $\begin{array}{l}\text { Classification } \\
\text { de Deer }\end{array}$ \\
\hline Diorite quartzique altéré & $\mathbf{4 0}$ & Faible \\
\hline
\end{tabular}

\begin{tabular}{|l|l|l|}
\hline fracturé + Chlorite & & \\
\hline $\begin{array}{l}\text { Caisse Minéralisée+ } \\
\text { chlorite }\end{array}$ & $\mathbf{3 6}$ & Faible \\
\hline Serpentine Franche & $\mathbf{2 6}$ & Faible \\
\hline
\end{tabular}

\subsubsection{Classification de Hoek et Brown GSI}

Introduit par Hoek (1995) et Hoek, Kaiser et Bawden [4] (1995), le GSI (Geological Strength Index) est un nombre sans dimension, déterminé empiriquement, qui varie entre 5 (très mauvaise qualité) et 85 (excellente qualité), et qui peut être estimé à partir d'un examen de la qualité de la masse rocheuse in situ. L'observation du terrain et l'étude des sondages carottés, ont permis de déterminer une valeur GSI moyenne approximative pour les différentes roches.

Le GSI modifié proposé par Sonmez et Ulusay (1999) [5], est un perfectionnement de la GSI estimait trop approximative.

Le calcul du GSI et GSI modifié pour le massif rocheux de la ST2 de Bou-azzer EST a permis de classer les différents faciès tableau 2.

Tableau 2. Classification GSI et GSI modifié

\begin{tabular}{|l|l|l|l|}
\hline Roche & GSI & $\begin{array}{l}\text { GSI } \\
\text { Modifié }\end{array}$ & Classification \\
\hline $\begin{array}{l}\text { Diorite } \\
\text { quartzique altéré } \\
\text { chloritisé fracturé }\end{array}$ & 65 & 43,00 & Moyenne \\
\hline $\begin{array}{l}\text { Caisse } \\
\text { Minéralisée+ } \\
\text { Chlorite }\end{array}$ & 65 & 42,50 & Moyenne \\
\hline Serpentine & 40 & 33,67 & Mauvaise \\
\hline
\end{tabular}

\subsubsection{Classification de l'AFTES [7]}

L'Association Française des Travaux en Souterrain (AFTES), précise clairement les divers facteurs influençant la conception d'un projet souterrain dans un massif rocheux.

Pour chaque critère, l'AFTES fournit des recommandations propres afin de permettre le choix du soutènement, après étude des trois faciès de la ST2 (-560) les recommandations sont regroupées dans le tableau 3

Tableau 3. Le soutènement retenu après l'utilisation de l'AFTES pour la ST2 (-560 m).

\begin{tabular}{|c|c|}
\hline Roche & Diorite quartzique altéré chloritisé fracturé \\
\hline $\begin{array}{l}\text { Les } \\
\text { Soutènements } \\
\text { données par } \\
\text { l'AFTES } \\
\end{array}$ & $\begin{array}{l}\text {-Le béton projeté } \\
\text { - Boulons à ancrage ponctuel } \\
\text { - Boulons à ancrage réparti } \\
\text { - Cintres lourds. }\end{array}$ \\
\hline $\begin{array}{l}\text { Diorite } \\
\text { quartzique } \\
\text { altéré }\end{array}$ & $\begin{array}{l}\text {-Le béton projeté } \\
\text { - Boulons à ancrage ponctuel } \\
\text { - Boulons à ancrage réparti }\end{array}$ \\
\hline
\end{tabular}




\begin{tabular}{|l|l|}
\hline $\begin{array}{l}\text { chloritisé } \\
\text { fracturé }\end{array}$ & - Cintres lourds. \\
\hline $\begin{array}{l}\text { Serpentine } \\
\text { franche }\end{array}$ & $\begin{array}{l}\text {-Le béton projeté } \\
\text {-Boulons à ancrage ponctuel } \\
\text {-Boulons à ancrage réparti }\end{array}$ \\
& $\begin{array}{l}\text {-Cintres lourds. } \\
\text {-Voussoirs constitués de plaques de métal } \\
\text { assemblées. }\end{array}$ \\
\hline $\begin{array}{l}\text { Caisse } \\
\text { minéralisée+ } \\
\text { chlorite }\end{array}$ & $\begin{array}{l}\text { - Le béton projeté } \\
\text { - Boulons à ancrage ponctuel } \\
\text { - Boulons à ancrage réparti } \\
\text { - Cintres lourds. }\end{array}$ \\
\hline
\end{tabular}

\subsubsection{Classification de Barton [8,9] :}

On utilisant la méthode de Barton ou Q-System nous avons pu classer le massif de la ST2 (-560) de mauvais à très mauvais et de proposer le soutènement adapté (tableau 4).

Tableau 4 : soutènement pour chaque faciès donné par le Qsystème.

\begin{tabular}{|l|l|l|l|l|}
\hline Roche & $\begin{array}{l}\text { Qsy } \\
\mathrm{s}\end{array}$ & $\begin{array}{l}\text { Classification } \\
\text { de Barton }\end{array}$ & $\begin{array}{l}\mathrm{D} \\
\mathrm{e}\end{array}$ & Soutènement \\
\hline $\begin{array}{l}\text { Diorite } \\
\text { quartzique } \\
\text { altéré } \\
\text { chloritisé } \\
\text { fracturé }\end{array}$ & 2,67 & Mauvaise & 2 & $\begin{array}{l}\text { Boulonnage } \\
\text { Lb=1,60m/Es=1, } \\
35 \mathrm{~m}\end{array}$ \\
\hline $\begin{array}{l}\text { Caisse } \\
\text { minéralisée } \\
+ \text { chlorite }\end{array}$ & 1,60 & Mauvaise & 2 & $\begin{array}{l}\text { Boulonnage } \\
\text { Lb=1,60m/Es=1, } \\
35 \mathrm{~m}\end{array}$ \\
\hline $\begin{array}{l}\text { Serpentine } \\
\text { nerét }\end{array}$ & 0,22 & $\begin{array}{l}\text { Très } \\
\text { Mauvaise }\end{array}$ & 2 & $\begin{array}{l}\text { Grillage + Béton } \\
\text { projeté 50 - 90 } \\
\text { mm avec } \\
\text { boulonnage }\end{array}$ \\
\hline
\end{tabular}

\subsubsection{Classification de Bieniawski [10, 11]}

La classification de Bieniawski ou la RMR est la somme de cinq notes représentant la quantification de cinq paramètres caractérisant le rocher (la résistance en compression simple; le RQD; espacement de famille des discontinuités; la rugosité et l'altération des discontinuités, la présence d'eau; l'orientation de la galerie par rapport à l'orientation de la famille de discontinuités critiques). La somme des cinq notes est ajustée par une note relative au sens du creusement par rapport au sens du pendage des discontinuités. Bieniawski, par cette classification, propose la méthode d'excavation envisageable et le type de soutènement le mieux adapté.

D'après la RMR, le massif de la ST2 (-560m) est classé de médiocre pour les Serpentines franches à moyen pour les Diorites quartziques altérés chloritisés fracturés et pour la caisse minéralisée et Chlorite, le soutènement proposé est de type Béton projeté et Cintres métalliques pour les trois type de facies et Boulon à ancrage uniquement pour les Diorites quartziques altérés chloritisés fracturés et la caisse minéralisée et Chlorite.

\section{Etude de la fracturation}

L'existence de plusieurs type de discontinuités (failles, diaclases et joints) ont rendu la réalisation de simulation numérique très difficile, ces discontinuités créent un réseau de fractures naturelles qui découpent les blocs en différentes formes susceptibles de se détacher ou de glisser dans l'excavation, favorisant ainsi l'infiltration des eaux et créant une pression sur le massif. Les études classiques entamées précédemment montrent leurs limites en pratique pour la pose du soutènement, parce qu'ils doivent prendre en considération les caractéristiques des discontinuités, d'où une analyse structurale du massif s'avère indispensable

Les levées de la fracturation ont été traitées par Dips (Logiciel de projection stéréographique sur le canevas de Wolf-Smith).

La projection stéréographique montre une large concentration des pôles vers l'Est (figure 4).

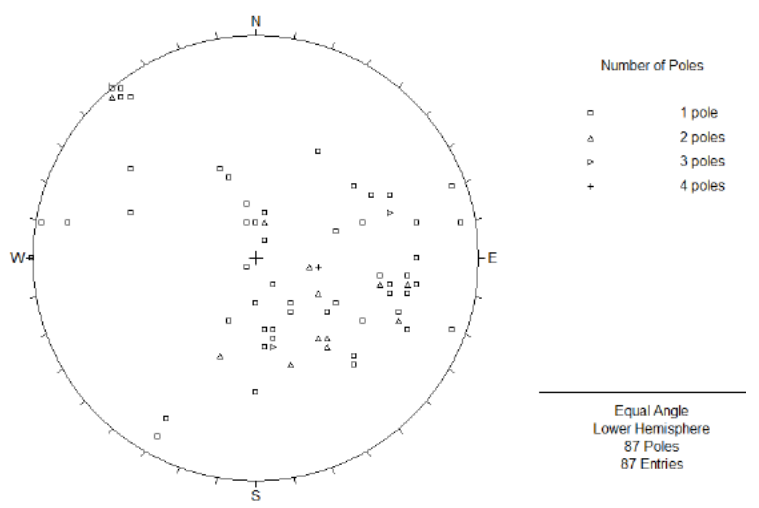

Fig 4: Distribution des pôles des discontinuités au niveau $-560 \mathrm{~m}$

Les discontinuités se répartissent autour de trois familles principales NNE-SSW, NNW-SSE, NE-SW et une famille secondaire NW-SE très diffuse, elle est de moindre importance) (figure 5).
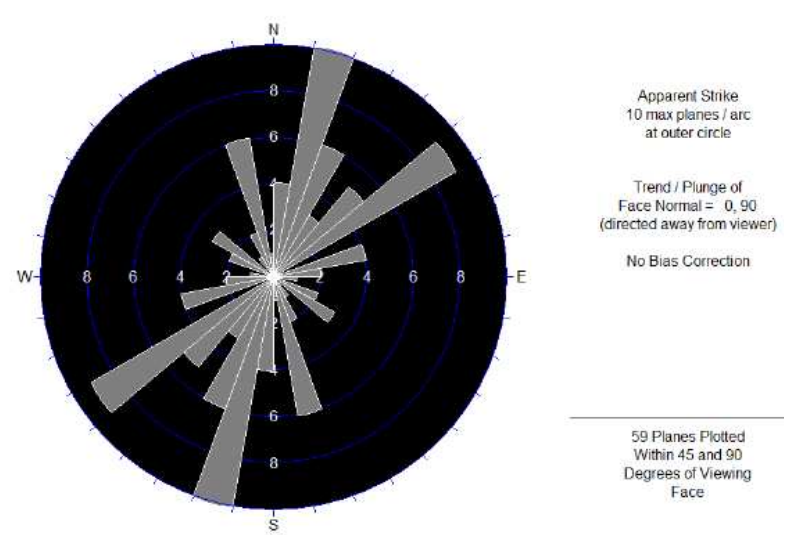

Fig 5 : Diagramme Rosette des lignes des discontinuités Niv$560 \mathrm{~m}$.

La concentration des pôles montre quatre à cinq zones de concentration avec une concentration maximale de $10.25 \%$. (figure 6) 

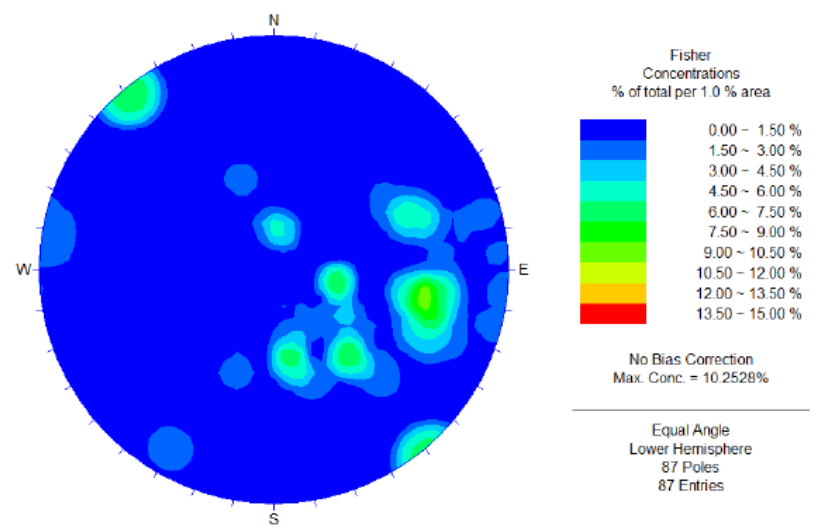

Fig 6: Contours des concentrations des pôles au niveau -560m

Le regroupement des pôles qui définissent la même famille de discontinuités (figure 7), nous a permis d'identifier trois familles principales avec des joints mineurs, de directions variable, et de pendages faible à moyen et subvertical (tableau 5)
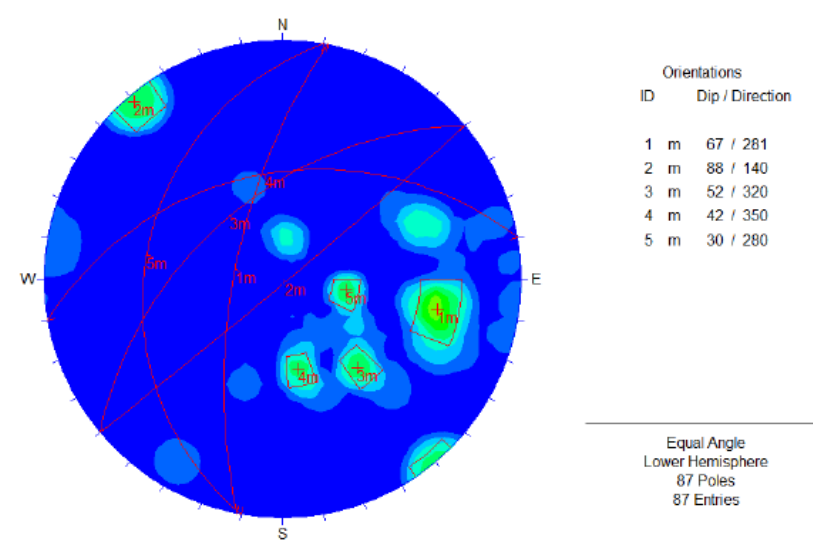

Fig 7: Regroupement des pole au niveau $-560 \mathrm{~m}$.

Tableau 5: Les principales familles de discontinuités au niveau $-560 \mathrm{~m}$

\begin{tabular}{|l|l|l|l|}
\hline Famille & Pendage & $\begin{array}{l}\text { Sens du } \\
\text { pendage }\end{array}$ & Description \\
\hline 1 & 67 & 283 & $\begin{array}{l}\text { Famille } \\
\text { principale }\end{array}$ \\
\hline 2 & 88 & 140 & $\begin{array}{l}\text { Famille } \\
\text { principale }\end{array}$ \\
\hline 3 & 52 & 320 & $\begin{array}{l}\text { Famille } \\
\text { principale }\end{array}$ \\
\hline 4 & 42 & 350 & $\begin{array}{l}\text { Joints } \\
\text { Mineurs }\end{array}$ \\
\hline 5 & 30 & 280 & $\begin{array}{l}\text { Joints } \\
\text { Mineurs }\end{array}$ \\
\hline
\end{tabular}

L'étude de la fracturation montre que le massif est caractérisé par un état de fracturation important, pour l'évaluation des risques engendrés par ces familles de discontinuités, nous avons utilisé les techniques Kinematics recommandés par Goodman, en ajoutant les variabilités standard 1 et 2 ainsi que les plans des épontes diorite et serpentine

\subsection{Risque au basculement :}

Nous avons considéré les deux épontes de la galerie, la Diorite Quartzique de pendage subvertical $\left(85^{\circ}\right)$ et de direction de pendage 160 et la Serpentine de pendage moyen $60^{\circ}$ et de direction de pendage 340 . Les plans limitant le risque de basculement sont caractérisés par les mêmes directions que les épontes, leurs pendages est calculé par la formule : $P L=|P-\varphi|$

Avec PL : pendage du plan limite, $\mathrm{P}$ : pendage du plan de l'éponte et $\varphi$ l'angle de frottement interne.

Cette analyse nous a permis de visualiser les zones de risques de chute de blocs, ainsi que les familles responsables de ces risques (figure 8), ainsi il y a un risque de basculement dans la partie entre la minéralisation et l'éponte de la serpentine engendré par la deuxième famille $(88 / 140)$.

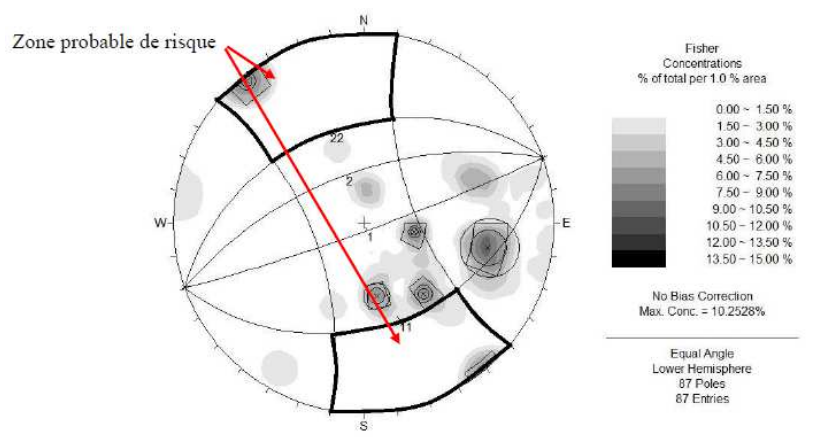

Fig 8 : Risque de Basculement.

\subsection{Risque de glissement.}

Nous avons considéré des enveloppes engendrées par les plans des deux épontes et des connes limites verticaux de direction N00 et d'angles égale à l'angle de frottement interne de la roche

Par cette analyse nous avons pu visualiser les zones de risques de glissement, ainsi que les familles responsables de ces risques (Figure 9)

Le risque de glissement est localisé dans la partie entre la minéralisation et la serpentine engendré par la famille principale 3 et à moindre degré par les familles 4 et 5 


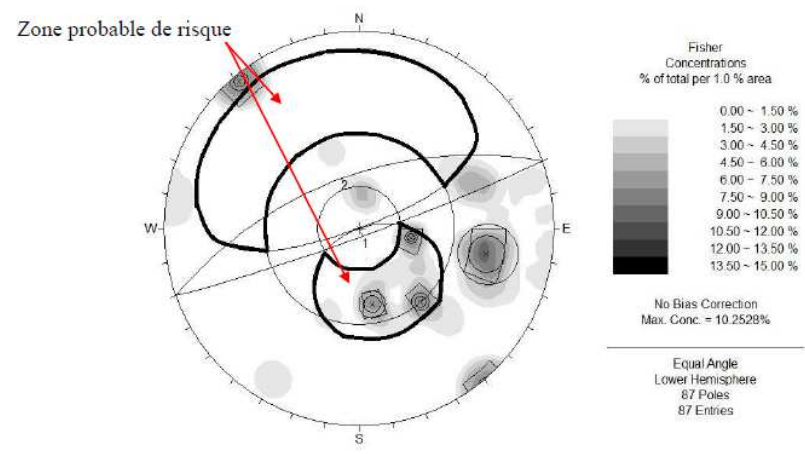

Fig 9 : Risque de glissement

Les risques engendrés par l'intersection des différentes familles de discontinuité est déterminé en tenant compte des plans des épontes et des plans des familles de discontinuités, et en ajoutant des connes verticaux de direction N00 d'angle égale à $90-\varphi \quad(\varphi$ : angle de frottement interne de la roche encaissante). Nous avons donc pu localiser les zones de risques d'intersections des familles de discontinuité, engendré par l'intersection des familles 1-3, 1-4, 5-4 et 4-3 dans la partie entre la minéralisation et la serpentine et 1-2 dans la partie entre la minéralisation et les diorites. Les familles 4 et 5 ne sont pas principales et constituent de ce fait un risque négligeable; la famille 2 qui caractérise les plans de schistosités des serpentines n'affecte pas les diorites qui se trouve épargnée du risque d'instabilité (Figure 10).

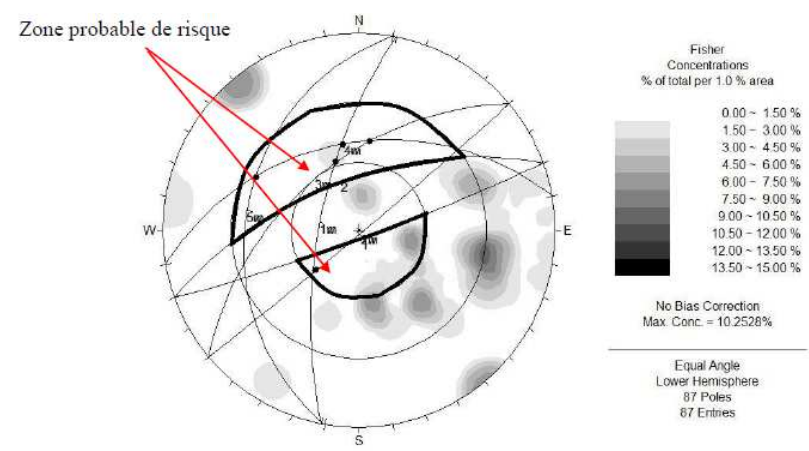

Fig 10: Risques de glissement engendrés par l'intersection des discontinuités.

\section{Conclusion}

Les filons minéralisé cobaltifères de la mine de Bouazzer se développent dans les serpentines au contact avec les diorites quartziques du Précambrien II inférieur.

La classification empirique (Deer, Hoek et Brown, Barton, Bienswiski et L'AFTES), du massif rocheux de la ST2 à Bou-Azzer Est, le qualifié de mauvais terrain et propose un soutènement de type Béton projeté et Cintres métalliques pour les trois type de facies Diorites quartziques altérés chloritisés fracturés, Serpentines et la caisse minéralisée et Chlorite et Boulon à ancrage avec précaution pour les Serpentines

L'analyse avancée de la fracturation du massif rocheux a permis le relevé des principales directions des discontinuités NNE-SSW, NNW-SSE, NE-SW, et de déterminer et de localiser les zones à risque de basculement et de glissement, puis de proposer un soutènement surfacique (Grillage) ou bien porteur (Cintres métalliques, Cadres en bois) pour soutenir les épontes fracturées et instable. Cependant l'étude des risques engendrés par l'intersection des discontinuités nous a permis de localiser les intersections des familles de discontinuités pour la mise en place du soutènement.

\section{Références et bibliographie}

[1] A Ait Bendra \& R Bkali, Exploitation des filons cobaltifères de Bou-Azzer au contact des serpentines franches (cas d'étude: Aghbar et Bou-Azzer Est),CTT, 2013.

[2] Leblanc, M., 1975. Ophiolites précambriennes et gites arséniés de Cobalt (Bou Azzer - Maroc), Université Paris VI, Paris.

[3] Deere, D.U. and Deere, D.W. 1988. The rock quality designation (RQD) index in practice. In Rock classification systems for engineering purposes, (ed. L. Kirkaldie), ASTM Special Publication 984, 91101. Philadelphia: Am. Soc. Test. Mat.

[4] Hoek, E., Kaiser, P.K. and Bawden. W.F. 1995. Support of underground excavations in hard rock. Rotterdam: Balkema.

[5] Sonmez, H., and Ulusay, R. (1999). Modification to the geological strength index (GSI) and their applicability to stability of slopes. International Journal of Rock Mechanics and Mining Science, 36: 743-760.

[6] A. Palmström, Journal of Rock Mechanics and Tunnelling Technology, The Rock Mass Index (RMi) applied in rock mechanics and rock engineering, 11, No.2, 1-40, (1996)

[7] AFTES : Recommandations du Groupe de Travail $\mathrm{n}^{\circ} 1$. Caractérisation des massifs rocheux utile à l'étude et à la réalisation des ouvrages souterrains. Tunnels et ouvrages souterrains, (177) :pp 138-170, mai-juin 2003.

[8] N. Barton, R. Lien, and J. Lunde, Engineering classification of rock masses for the design of tunnel support, (1974)

[9] N. Barton, International Journal of Rock Mechanics \& Mining Sciences, Some new Q-value correlations to assist site characteristics and tunnel design, 185-216, (2002)

[10] Z.T. Bieniawski, Rock Mechanics Design in Mining and Tunnelling, 272,(1984)

[11] Z.T. Bieniawski, Classification of Rock Masses for Engineering: The RMR System and Future Trends, Comprehensive rock engineering, 3,522-542, (1993) 